\title{
An Adiabatic Theorem Applicable to the Stark Effect
}

\author{
E. B. Davies \\ Department of Mathematics, King's College, Strand, London WC2R 2LS, England
}

\begin{abstract}
We prove an adiabatic theorem applicable to an atom evolving in a slowly varying electric field. This yields an operational estimate of the tunneling rate even for systems where complex scaling techniques are not applicable.
\end{abstract}

\section{Introduction}

The discovery of the spectral theorem by Stone and von Neumann in 1930 provided an extremely powerful tool for early quantum mechanics, and enabled a deep investigation both of bound states and of scattering theory to be initiated. At the computational level these have been well understood for many years, and their theoretical analysis is now largely complete. On the other hand the study of metastable states (resonances) has made much slower progress, and it is only with the introduction in the last decade of ideas known collectively as complex scaling $([1,2,4,5,12,13]$ and references there in) that real insight has been achieved.

A standard application of complex scaling is to the Stark effect $[4,5,13]$ for the hydrogen atom, with Hamiltonian

$$
H_{E}=-\Delta-\frac{2}{|x|}+E x_{3}
$$

Although this operator is known to have no bound states for $E \neq 0$, complex scaling leads to the discovery of a complex eigen value $\lambda_{E}$ whose associated eigenfunction $\phi_{E}$ is not square-integrable. The real part of $\lambda_{E}$ is often identified as the perturbed energy level of the hydrogen atom, while the imaginary part, which is exponentially small, is identified as the decay or tunneling rate.

One way of trying to justify these identifications operationally would be to consider the evolution of the ground state of the hydrogen atom if the external field is switched on adiabatically. Thus one examines the form of the solution of $\psi^{\prime}(t)=$ $-i H_{E(t)} \psi(t)$ if $E(0)=0$, and $E(t)$ varies very slowly. The adiabatic theorem of Kato [6] is not applicable here because $H_{E}$ has no bound states for $E \neq 0$. The obvious guess that $\psi(t)$ should be near to the resonance eigenfunction $\phi_{E(t)}$ up to a phase is clearly also impossible if one measures nearness in $L^{2}$ norm. We do not attempt to 
prove such an approximation for some other appropriate norm, but point out that one might enquire about the operational significance of any other norm which is used.

We next comment that the idea of obtaining a metastable state by switching on the field slowly enough is not entirely correct. The difficulty is that tunneling allows the perturbed ground state to dissipate very slowly. Thus the rate of increase of the field strength must be small enough to avoid impulsive reactions, but large enough to render tunneling effects unimportant. This feature of having to keep between two very different time scales is typical of metastable phenomena, and is what makes their rigorous analysis so fascinating.

The basic ingredient of our approach is the construction of a particular family of states $\phi_{E}$ and scalars $\lambda_{E}$ such that

(i) $\left\|\phi_{E}\right\|=1$ all $E$,

(ii) $E \rightarrow \phi_{E}$ and $E \rightarrow \lambda_{E}$ are smooth functions of $E$,

(iii) $\left\|H_{E} \phi_{E}-\lambda_{E} \phi_{E}\right\| \leqq \varepsilon(E)$, where $\varepsilon(E) \rightarrow 0$ rapidly as $E \rightarrow 0$.

These requirements are closely related to ideas of spectral concentration $[3,7,11]$. In the standard adiabatic theorem [6] one takes $\phi_{E}$ and $\lambda_{E}$ to be an eigenstate and corresponding eigenvalue of $H_{E}$, so that $\varepsilon(E)=0$, and one uses perturbation theory to prove (ii).

In the case of the Stark effect one could define $\phi_{E}$ to be a truncation of the resonance eigenfunction to a region near the origin [4, p. 870], and then define $\lambda_{E}$ to be the real part of the resonance eigenvalue. Although the particular truncation chosen is not crucial within certain limits, the lack of any canonical choice makes it difficult to establish a precise connection between the decay rate and the imaginary part of the resonance eigenvalue [4, p. 857]. We shall in fact make a slightly different choice of $\phi_{E}$, which should have similar numerical properties.

Although we shall for simplicity write down our entire theory for the hydrogen atom, we do not see any difficulty in extending our method to multi-electron systems with several fixed nuclei. One can certainly replace the Coulomb potential $V(x)=$ $-2 /|x|$ by an arbitrary $V \in L^{2}+\left(L^{\infty}\right)_{\varepsilon}$ with no essential difficulties. Such potentials may not have any of the dilation properties needed for complex scaling, so that there is no obvious possibility in general of defining $\phi_{E}$ as a truncation of a resonance eigenfunction. In spite of this we are able to obtain a lower bound on the decay rate which is of the same general form as that given by the Oppenheimer formula [4]. Our main result is Theorem 12, but its application to adiabatic problems is more easily seen in Corollary 13.

\section{The Modified Hamiltonian}

Our concern will be to study the solution of the time-dependent evolution equation

$$
\psi^{\prime}(t)=-i\left(-\Delta-\frac{2}{|x|}+E(t) x_{3}\right) \psi(t)
$$


in $L^{2}\left(\mathbb{R}^{3}\right)$, when $E(t)$ is a slowly varying, twice continuously differentiable, bounded function with $E(0)=0$, and when $\psi(0)$ equals the ground state eigenfunction $\phi_{0}$ of the zero field hydrogen atom. Because the time-dependent part of (1) is rather singular we shall however consider a truncated version of this problem. Namely we define $\psi(t)$ to be the solution of

$$
\begin{aligned}
\psi^{\prime}(t) & =-i\left(H_{0}+E(t) V\right) \psi(t) \\
& \equiv-i H_{E(t)} \psi(t),
\end{aligned}
$$

where

$$
H_{0}=-\Delta-\frac{2}{|x|}
$$

and

$$
V(x)=\left\{\begin{array}{l}
x_{3} \text { if }\left|x_{3}\right| \leqq N \\
N \operatorname{sign}\left(x_{3}\right) \text { if }\left|x_{3}\right|>N,
\end{array}\right.
$$

and $N$ is a very large constant.

One can argue [11, p. 46] that (2) is just as relevant physically for the description of the Stark effect as is (1). We have, however, only made the truncation to simplify domain considerations. It will be seen that our estimates are all independent of $N$ provided $N$ is large enough, so that one might take the limit $N \rightarrow \infty$ at the end of all the calculations of this paper. This would require one first to prove that the solution $\psi_{N}(t)$ of (2) converges to the solution $\psi_{\infty}(t)$ of $(1)$, which we regard as a separate problem. We shall not mention again the $N$-dependence of $\psi(t)$. We quote from [9; 10, p. 290] the theorem which ensures the existence of a solution of (2).

Proposition 1. If $E(t)$ is twice continuously differentiable and $\mathscr{D}$ is the domain of $\mathrm{H}_{0}$, then there is a family $U_{t}$ of unitary operators on $L^{2}\left(\mathbb{R}^{3}\right)$ such that if $\psi(t)=U_{t} \psi(0)$, where $\psi(0) \in \mathscr{D}$, then $\psi(t) \in \mathscr{D}$ for all $t \geqq 0$ and $\psi^{\prime}(t)=-i H_{E(t)} \psi(t)$.

We shall compare $\psi(t)$ with the ground state of a Hamiltonian

$$
K_{E}=H_{0}+E W,
$$

where $E=E(t)$ and the external field is modified so as to prevent the possibility of tunneling. If

$$
\delta=\sup \{|E(t)|: 0 \leqq t<\infty\},
$$

then the smallest tunneling length as $t$ varies is $\delta^{-1}$, so $W$ should coincide with $V$ in the region where $|x|=o\left(\delta^{-1}\right)$. We shall in fact put

$$
W(x)=\left\{\begin{array}{l}
x_{3} \text { if }\left|x_{3}\right| \leqq 1 / 8 \delta \\
\frac{1}{8 \delta} \operatorname{sign} x_{3} \text { if }\left|x_{3}\right|>1 / 8 \delta .
\end{array}\right.
$$

We also put $A=V-W$, and note for later use that

$$
\left\|A e^{-\beta\left|Q_{3}\right|}\right\| \leqq \beta^{-1} e^{-\beta / 8 \delta} .
$$


The rest of this section is of a technical nature and may be omitted at a first reading. We wish to obtain estimates of various quantities involving the ground state energy $\lambda_{E}$ and the ground state eigenfunction $\phi_{E}$ of $K_{E}$. The point will be to find the dependence of these quantities upon $\delta$, uniformly for all $E$ in the interval $[-\delta, \delta]$. We assume this restriction upon $E$ throughout.

Lemma 2. We have

$$
\operatorname{Sp}\left(K_{E}\right) \subseteq\left\{\lambda_{E}\right\} \cup\left[-\frac{3}{8}, \infty\right),
$$

where the ground state eigenvalue $\lambda_{E}$ is non-degenerate and satisfies $-\frac{9}{8} \leqq \lambda_{E} \leqq-1$.

Proof. It is a standard fact that

$$
\operatorname{Sp}\left(H_{0}\right) \subseteq\{-1\} \cup\left[-\frac{1}{4}, \infty\right),
$$

so the lemma follows by combining standard variational estimates with the bound $\|E W\| \leqq \frac{1}{8}$ and the identity $\left\langle W \varphi_{0}, \varphi_{0}\right\rangle=0$.

It is well known that the ground state wave function $\phi_{E}$ is non-degenerate and strictly positive, and we always normalize it by $\left\|\phi_{E}\right\|=1$. Perturbation theory shows that $\phi_{E}$ and $\lambda_{E}$ are real analytic functions of $E$. We shall make frequent use of the formula

$$
P_{E} \equiv\left|\phi_{E}\right\rangle\left\langle\phi_{E}\right|=\frac{1}{2 \pi i} \int_{\gamma}\left(z-K_{E}\right)^{-1} d z
$$

where $\gamma$ is any closed contour containing $\left[-\frac{9}{8},-1\right]$ and not meeting $\left[-\frac{3}{8}, \infty\right)$.

Lemma 3. We have

$$
\left\|\phi_{E}^{\prime}\right\| \leqq\left\|P_{E}^{\prime}\right\|, \quad\left\|\phi_{E}^{\prime \prime}\right\| \leqq\left\|P_{E}^{\prime \prime}\right\|
$$

Proof. Since $\left\langle\phi_{E}^{\prime}, \phi_{E}\right\rangle=0$ and $P_{E}^{\prime}=\left|\phi_{E}^{\prime}\right\rangle\left\langle\phi_{E}|+| \phi_{E}\right\rangle\left\langle\phi_{E}^{\prime}\right|$, we see that $\left\|\phi_{E}^{\prime}\right\|=\left\|P_{E}^{\prime} \phi_{E}\right\| \leqq\left\|P_{E}^{\prime}\right\|$. Secondly

$$
P_{E}^{\prime \prime}=\left|\phi_{E}^{\prime \prime}\right\rangle\left\langle\phi_{E}|+2| \phi_{E}^{\prime}\right\rangle\left\langle\phi_{E}^{\prime}|+| \phi_{E}\right\rangle\left\langle\phi_{E}^{\prime \prime}\right|
$$

so $\left\langle P_{E}^{\prime \prime} \phi_{E}, \phi_{E}\right\rangle=2\left\langle\phi_{E}^{\prime \prime}, \phi_{E}\right\rangle$, while $P_{E}^{\prime \prime} \phi_{E}=\phi_{E}^{\prime \prime}+\phi_{E}\left\langle\phi_{E}^{\prime \prime}, \phi_{E}\right\rangle$.

Therefore $\phi_{E}^{\prime \prime}=P_{E}^{\prime \prime} \phi_{E}-\frac{1}{2}\left\langle P_{E}^{\prime \prime} \phi_{E}, \phi_{E}\right\rangle \phi_{E}$, so

$$
\begin{aligned}
\left\|\phi_{E}^{\prime \prime}\right\|^{2} & =\left\|P_{E}^{\prime \prime} \phi_{E}\right\|^{2}-\left\langle P_{E}^{\prime \prime} \phi_{E}, \phi_{E}\right\rangle^{2}+\frac{1}{4}\left\langle P_{E}^{\prime \prime} \phi_{E}, \phi_{E}\right\rangle^{2} \\
& \leqq\left\|P_{E}^{\prime \prime} \phi_{E}\right\|^{2} \leqq\left\|P_{E}^{\prime \prime}\right\|^{2}
\end{aligned}
$$

Lemma 4. We have

$$
\left\|\phi_{E}^{\prime}\right\| \leqq \frac{1}{4 \delta}, \quad\left\|\phi_{E}^{\prime \prime}\right\| \leqq \frac{1}{4 \delta^{2}},\left\langle\phi_{E}, \phi_{0}\right\rangle \geqq \frac{3}{4} .
$$


Proof. Use of the resolvent identities enables one to obtain

$$
P_{E}^{\prime}=\frac{1}{2 \pi i} \int_{\gamma}\left(z-K_{E}\right)^{-1} W\left(z-K_{E}\right)^{-1} d z
$$

from (4). Deforming $\gamma$ into the contour $\gamma(t)=-\frac{3}{4}+i t$, we see from Lemma 2 that

$$
\left\|\left(\gamma(t)-K_{E}\right)^{-1}\right\| \leqq\left(t^{2}+\frac{1}{16}\right)^{-1 / 2}
$$

for all $t$. Thus

$$
\begin{aligned}
\left\|P_{E}^{\prime}\right\| & \leqq \frac{1}{2 \pi} \int_{-\infty}^{\infty} \frac{1}{8 \delta}\left(t^{2}+\frac{1}{16}\right)^{-1} d t \\
& =\frac{1}{4 \delta},
\end{aligned}
$$

which yields the bound on $\left\|\phi_{E}^{\prime}\right\|$ by Lemma 3. The bound on $\left\|\phi_{E}^{\prime \prime}\right\|$ is obtained similarly from the identity

$$
P_{E}^{\prime \prime}=\frac{2}{2 \pi i} \int_{\gamma}\left(z-K_{E}\right)^{-1} W\left(z-K_{E}\right)^{-1} W\left(z-K_{E}\right)^{-1} d z .
$$

Finally

$$
\begin{aligned}
\left|\left\langle\phi_{E}, \phi_{0}\right\rangle-1\right| & \leqq \int_{0}^{E}\left|\left\langle\phi_{F}^{\prime}, \phi_{0}\right\rangle\right| d F \\
& \leqq \frac{E}{4 \delta} \leqq \frac{1}{4}
\end{aligned}
$$

which implies (5).

We next define the reduced resolvent operator $S_{E}$ by

$$
S_{E}=\left(\lambda_{E}-K_{E}\right)^{-1}\left(1-P_{E}\right) .
$$

Lemma 5. We have $\left\|S_{E}\right\| \leqq 2, \quad\left\|S_{E}^{\prime}\right\| \leqq 6 / \delta$.

Proof. The first estimate is a consequence of Lemma 2 and the spectral theorem. For the second we use the alternative definition

$$
S_{E}=\frac{1}{2 \pi i} \int_{\gamma}\left(z-\lambda_{E}\right)^{-1}\left(z-K_{E}\right)^{-1} d z,
$$

where $\gamma$ is the circle $\left\{z:|z+1|=\frac{3}{8}\right\}$ so that

$$
\left\|\left(z-K_{E}\right)^{-1}\right\| \leqq 4
$$


for all $z$ on $\gamma$ and all $|E| \leqq \delta$. Differentiating (7) we obtain

$$
\begin{aligned}
S_{E}^{\prime}= & \frac{1}{2 \pi i} \int_{\gamma} \lambda_{E}^{\prime}\left(z-\lambda_{E}\right)^{-2}\left(z-K_{E}\right)^{-1} d z \\
& +\frac{1}{2 \pi i} \int_{\gamma}\left(z-\lambda_{E}\right)^{-1}\left(z-K_{E}\right)^{-1} W\left(z-K_{E}\right)^{-1} d z .
\end{aligned}
$$

Using (8) and the estimate $\left|\lambda_{E}^{\prime}\right|=\left|\left\langle W \phi_{E}, \phi_{E}\right\rangle\right| \leqq\|W\|=\frac{1}{8 \delta}$ yields

$$
\begin{gathered}
\left\|S_{E}^{\prime}\right\| \leqq \frac{1}{2 \pi} \cdot \frac{1}{8 \delta} \cdot 4^{3} \cdot 2 \pi \cdot \frac{3}{8}+\frac{1}{2 \pi} \cdot 4^{2} \cdot \frac{1}{8 \delta} \cdot 4 \cdot 2 \pi \cdot \frac{3}{8} \\
=6 / \delta .
\end{gathered}
$$

Our next task is to prove the exponential decay of certain wave-functions. While this could be done by JWKB methods, we prefer to use an idea of O'Connor $[8 ; 11$, p. 196]. The crucial idea is to investigate the spectral properties of the operator

$$
\begin{aligned}
K_{E, \beta} & =e^{\beta Q_{3}}\left(H_{0}+E W\right) e^{-\beta Q_{3}} \\
& =H_{0}+E W+2 \beta \frac{\partial}{\partial x_{3}}-\beta^{2}
\end{aligned}
$$

for small complex $\beta$, noting that $K_{E, \beta}$ is unitarily equivalent in an obvious manner to $K_{E}$ if $\beta$ is purely imaginary.

Lemma 6. There exists an absolute constant c such that if $-\delta \leqq E \leqq \delta$ and $|\beta| \leqq c$ and $z$ lies on the circle $\gamma=\left\{z:|z+1|=\frac{3}{8}\right\}$, then $\left\|\left(z-K_{E, \beta}\right)^{-1}\right\| \leqq \frac{16}{3}$.

Proof. We write

$$
\begin{aligned}
\left(z-K_{E, \beta}\right)\left(z-H_{0}\right)^{-1} & =1-E W\left(z-H_{0}\right)^{-1}-\left(2 \beta \frac{\partial}{\partial x_{3}}-\beta^{2}\right)\left(z-H_{0}\right)^{-1} \\
& =1+D \quad \text { (say). }
\end{aligned}
$$

Now $\|E W\| \leqq \frac{1}{8}$ and $\left\|\left(z-H_{0}\right)^{-1}\right\| \leqq \frac{8}{3}$ for all $z$ on $\gamma$, so $\left\|E W\left(z-H_{0}\right)^{-1}\right\| \leqq \frac{1}{3}$. If $|\beta| \leqq \frac{1}{5}$, then

$$
\begin{aligned}
\left\|\left(2 \beta \frac{\partial}{\partial x_{3}}-\beta^{2}\right)\left(z-H_{0}\right)^{-1}\right\| & \leqq 2|\beta|\left\|\frac{\partial}{\partial x_{3}}\left(z-H_{0}\right)^{-1}\right\|+\frac{1}{5}|\beta|\left\|\left(z-H_{0}\right)^{-1}\right\| \\
& \leqq c_{1}|\beta|
\end{aligned}
$$

for some absolute constant $c_{1}$ and all $z$ on $\gamma$. Putting

$$
c=\min \left(\frac{1}{5}, \frac{1}{6 c_{1}}\right) \text {, }
$$


we see that $|\beta| \leq c$ implies

$$
\left\|\left(2 \beta \frac{\partial}{\partial x_{3}}-\beta^{2}\right)\left(z-H_{0}\right)^{-1}\right\| \leqq \frac{1}{6}
$$

so $\|D\| \leqq \frac{1}{2}$. This implies that

$$
\left\|\left(z-H_{0}\right)\left(z-K_{E, \beta}\right)^{-1}\right\| \leqq\left(1-\frac{1}{2}\right)^{-1}=2,
$$

so

$$
\left\|\left(z-K_{E, \beta}\right)^{-1}\right\| \leqq 2\left\|\left(z-H_{0}\right)^{-1}\right\| \leqq \frac{16}{3} .
$$

Lemma 7. If $-\delta \leqq E \leqq \delta$ and $|\beta| \leqq c$, then

$$
\begin{aligned}
& \left\|e^{\beta Q_{3}} P_{E} e^{-\beta Q_{3}}\right\| \leqq 2 \\
& \left\|e^{\beta Q_{3}} P_{E}^{\prime} e^{-\beta Q_{3}}\right\| \leqq 4 / 3 \delta, \\
& \left\|e^{\beta Q_{3}} S_{E} e^{-\beta Q_{3}}\right\| \leqq 8
\end{aligned}
$$

Proof. By following the method of O'Connor's lemma [11, p. 196] we deduce from (4) that

$$
e^{\beta Q_{3}} P_{E} e^{-\beta Q_{3}}=\frac{1}{2 \pi i} \int_{\gamma}\left(z-K_{E, \beta}\right)^{-1} d z,
$$

which may be estimated using Lemma 6 to yield (10). The other bounds follow from the formulae

$$
e^{\beta Q_{3}} P_{E}^{\prime} e^{-\beta Q_{3}}=\frac{1}{2 \pi i} \int_{\gamma}\left(z-K_{E, \beta}\right)^{-1} W\left(z-K_{E, \beta}\right)^{-1} d z
$$

and

$$
e^{\beta Q_{3}} S_{E} e^{-\beta Q_{3}}=\frac{1}{2 \pi i} \int_{\gamma}\left(z-\lambda_{E}\right)^{-1}\left(z-K_{E, \beta}\right)^{-1} d z .
$$

Lemma 8. If

$$
\Omega(x)=\left(\frac{4}{5}\right)^{3 / 2} \pi^{-1 / 2} e^{-4|x| / 5},
$$

then $\|\Omega\|=1$ and

$$
\left\langle\phi_{E}, e^{\beta Q_{3}} \Omega\right\rangle \geqq \frac{1}{2},
$$

provided $-\delta \leqq E \leqq \delta$ and $-\frac{1}{5} \leqq \beta \leqq \frac{1}{5}$.

Proof. Since $\phi_{0}(x)=\pi^{-1 / 2} e^{-|x|}$, we see that

$$
e^{\beta x_{3}} \Omega(x) \geqq\left(\frac{4}{5}\right)^{3 / 2} \phi_{0}(x)
$$

for all $x$, provided $-\frac{1}{5} \leqq \beta \leqq \frac{1}{5}$. The result follows by using (5) and the positivity of $\phi_{E}$. 
Lemma 9. If $-\delta \leqq E \leqq \delta$ and $c$ is defined by (9) then

$$
\left\|e^{ \pm c Q_{3}} \phi_{E}\right\| \leqq 4, \quad\left\|e^{ \pm c Q_{3}} \phi_{E}^{\prime}\right\| \leqq 6 / \delta .
$$

Proof.

$$
e^{ \pm c Q_{3}} P_{E} e^{\mp c Q_{3}} \Omega=e^{\mp c Q_{3}} \phi_{E}\left\langle\phi_{E}, e^{ \pm c Q_{3}} \Omega\right\rangle,
$$

so

$$
\begin{aligned}
\left\|e^{ \pm c Q_{3}} \phi_{E}\right\| & \leqq\left\langle\phi_{E}, e^{ \pm c Q_{3}} \Omega\right\rangle^{-1}\left\|e^{ \pm c Q_{3}} P_{E} e^{\mp c Q_{3}}\right\| \\
& \leqq 2.2=4
\end{aligned}
$$

by Lemmas 7 and 8 . Secondly

$$
e^{ \pm c Q_{3}} P_{E}^{\prime} e^{\mp c Q_{3}} \cdot e^{ \pm c Q_{3}} P_{E} e^{\mp c Q_{3}} \Omega=e^{ \pm c Q_{3}} \phi_{E}^{\prime}\left\langle\phi_{E}, e^{ \pm c Q_{3}} \Omega\right\rangle,
$$

so

$$
\begin{aligned}
\left\|e^{ \pm c Q_{3}} \phi_{E}^{\prime}\right\| & \leqq\left\langle\phi_{E}, e^{ \pm c Q_{3}} \Omega\right\rangle^{-1}\left\|e^{\mp c Q_{3}} P_{E}^{\prime} e^{ \pm c Q_{3}}\right\| \quad\left\|e^{ \pm c Q_{3}} P_{E} e^{\mp c Q_{3}}\right\| \\
& \leqq 2 \cdot \frac{4}{3 \delta} \cdot 2 \leqq 6 / \delta .
\end{aligned}
$$

\section{The Main Theorems}

In the last section we proved that various operator-valued functions of $E$ were norm differentiable, and obtained bounds on their derivatives in terms of $\delta$ under the assumption that $-\delta \leqq E \leqq \delta$. In this section we use these bounds to show that if $\psi(t)$ is the solution of (2) and $\theta(t)$ is defined by

$$
\theta(t)=\int_{0}^{t} \lambda_{E(S)} d s
$$

then $\left\|\psi(t)-e^{-i \theta(t)} \phi_{E(t)}\right\|$ remains small for very long times $t$, provided $E(t)$ varies slowly.

Lemma 10. If $F(\eta, t)$ is defined by

$$
F(\eta, t)=\left\langle\phi_{E(t)}, e^{i \theta(t)} U_{t} \eta\right\rangle
$$

for all $\eta$ in the set $\mathscr{C}=\{\eta \in \operatorname{Dom}(\Delta):\|\eta\| \leqq 1\}$, then

$$
\left\|\psi(t)-e^{-i \theta(t)} \phi_{E(t)}\right\|=\sup \{|F(\eta, t)-F(\eta, 0)|: \eta \in \mathscr{C}\} .
$$

Proof. We first observe that

$$
\begin{aligned}
\left\|\psi(t)-e^{-i \theta(t)} \phi_{E(t)}\right\| & =\left\|\phi_{0}-e^{-i \theta(t)} U_{t}^{*} \phi_{E(t)}\right\| \\
& =\|\xi(t)-\xi(0)\| \\
& =\sup \{|\langle\xi(t), \eta\rangle-\langle\xi(0), \eta\rangle|: \eta \in \mathscr{C}\},
\end{aligned}
$$

where $\xi(t)=e^{-i \theta(t)} U_{t}^{*} \phi_{E(t)}$. The estimate follows by observing that $F(\eta, t)=\langle\xi(t), \eta\rangle$. 
We may differentiate freely at various places in lemmas below because all the operators $H_{0}, H_{E}, K_{E}, K_{E, \beta}$ have the same domain $\mathscr{D}$, and this domain is invariant under $U_{t}$ by Proposition 1. Other operator derivatives exist by the arguments of Sect. 2, and we shall therefore concentrate on the bounds rather than the validity of every step below.

Lemma 11. If

$$
G(\eta, t)=i\left\langle E^{\prime}(t) S_{E(t)} \phi_{E(t)}^{\prime}, e^{i \theta(t)} U_{t} \eta\right\rangle,
$$

then

$$
|G(\eta, t)| \leqq\left|E^{\prime}(t)\right| / 2 \delta,
$$

and

$$
\begin{gathered}
\left|\frac{\partial}{\partial t}(F(\eta, t)-G(\eta, t))\right| \leqq \frac{\delta}{c} e^{-c / 8 \delta}\left\|e^{c\left|Q_{3}\right|} \phi_{E(t)}\right\|+\left\|\frac{\partial}{\partial t}\left(E^{\prime}(t) S_{E(t)} \phi_{E(t)}^{\prime}\right)\right\| \\
+\frac{\delta}{c}\left|E^{\prime}(t)\right| e^{-c / 8 \delta}\left\|e^{c\left|Q_{3}\right|} S_{E(t)} \phi_{E(t)}^{\prime}\right\| .
\end{gathered}
$$

Proof. The estimate (13) follows immediately from Lemmas 4 and 5. Also

$$
\begin{aligned}
\frac{\partial}{\partial t}(F(\eta, t) & -G(\eta, t))= \\
=E^{\prime}(t) & \left\langle\phi_{E(t)}^{\prime}, e^{i \theta(t)} U_{t} \eta\right\rangle-i\left\langle\phi_{E(t)},\left(\lambda_{E}(t)-H_{E(t)}\right) e^{i \theta(t)} U_{t} \eta\right\rangle \\
& -i\left\langle\frac{\partial}{\partial t}\left(E^{\prime}(t) S_{E(t)} \phi_{E(t)}^{\prime}\right), e^{i \theta(t)} U_{t} \eta\right\rangle \\
- & \left\langle E^{\prime}(t) S_{E(t)} \phi_{E(t)}^{\prime},\left(\lambda_{E(t)}-H_{E(t)}\right) e^{i \theta(t)} U_{t} \eta\right\rangle \\
=E^{\prime}(t) & \left\langle\phi_{E(t)}^{\prime}, e^{i \theta(t)} U_{t} \eta\right\rangle+i\left\langle E(t) A \phi_{E(t)}, e^{i \theta(t)} U_{t} \eta\right\rangle \\
& -i\left\langle\frac{\partial}{\partial t}\left(E^{\prime}(t) S_{E(t)} \phi_{E(t)}^{\prime}\right), e^{i \theta(t)} U_{t} \eta\right\rangle \\
& -\left\langle E^{\prime}(t)\left(1-P_{E(t)}\right) \phi_{E(t)}^{\prime}, e^{i \theta(t)} U_{t} \eta\right\rangle \\
& +\left\langle E^{\prime}(t) E(t) A S_{E(t)} \phi_{E(t)}^{\prime}, e^{i \theta(t)} U_{t} \eta\right\rangle .
\end{aligned}
$$

But the identity $\left\langle\phi_{E}^{\prime}, \phi_{E}\right\rangle=0$ implies that $\left(1-P_{E}\right) \phi_{E}^{\prime}=\phi_{E}^{\prime}$, so

$$
\begin{aligned}
& \left|\frac{\partial}{\partial t}(F(\eta, t)-G(\eta, t))\right| \\
& \quad \leqq\left\|E(t) A \phi_{E(t)}\right\|+\left\|\frac{\partial}{\partial t}\left(E^{\prime}(t) S_{E(t)} \phi_{E(t)}^{\prime}\right)\right\|+\left\|E^{\prime}(t) E(t) A S_{E(t)} \phi_{E(t)}^{\prime}\right\| \\
& \quad \leqq \frac{\delta}{c} e^{-c / 8 \delta}\left\|e^{c\left|Q_{3}\right|} \phi_{E(t)}\right\|+\left\|\frac{\partial}{\partial t}\left(E^{\prime}(t) S_{E(t)} \phi_{E(t)}^{\prime}\right)\right\|+\frac{\delta}{c}\left|E^{\prime}(t)\right| e^{-c / 8 \delta}\left\|e^{c\left|Q_{3}\right|} S_{E(t)} \phi_{E(t)}^{\prime}\right\|
\end{aligned}
$$

by (3) 
We can now put all our estimates together into the main theorem of the paper.

Theorem 12. There exists an absolute constant $c$ such that

$$
\begin{aligned}
\| \psi(t) & -e^{-i \theta(t)} \phi_{E(t)} \| \leqq \frac{8 \delta}{c} e^{-c / 8 \delta} t+\frac{1}{2}\left|E^{\prime}(t) / \delta\right|+\frac{1}{2}\left|E^{\prime}(0) / \delta\right| \\
& +\frac{1}{2} \int_{0}^{t}\left|E^{\prime \prime}(s) / \delta\right| d s+2 \int_{0}^{t}\left|E^{\prime}(s) / \delta\right|^{2} d s+\frac{96 \delta}{c} e^{-c / 8 \delta} \int_{0}^{t}\left|E^{\prime}(s) / \delta\right| d s
\end{aligned}
$$

for all $t \geqq 0$.

Proof. We first estimate the three terms on the right-hand side of (14). Since

$$
\left\|e^{c\left|Q_{3}\right|} \phi_{E(t)}\right\| \leqq\left\|e^{c Q_{3}} \phi_{E(t)}\right\|+\left\|e^{-c Q_{3}} \phi_{E(t)}\right\|,
$$

Lemma 9 establishes that the first term is dominated by $(8 \delta / c) e^{-c / 8 \delta}$. Differentiating the interior of the second term, Lemmas 4 and 5 establish that it is dominated by

$$
\begin{aligned}
& \left|E^{\prime \prime}(t)\right| \cdot 2 \cdot \frac{1}{4 \delta}+\left|E^{\prime}(t)\right|^{2}\left(\frac{6}{\delta} \cdot \frac{1}{4 \delta}+2 \cdot \frac{1}{4 \delta^{2}}\right) \\
& =\left|E^{\prime \prime}(t)\right| / 2 \delta+2\left|E^{\prime}(t)\right|^{2} / \delta^{2} .
\end{aligned}
$$

Finally the third term is dominated by

$$
\begin{aligned}
& \frac{\delta}{c}\left|E^{\prime}(t)\right| e^{-c / 8 \delta}\left\|e^{c Q_{3}} S_{E(t)} e^{-c Q_{3}}\right\| \quad\left\|e^{c Q_{3}} \phi_{E(t)}^{\prime}\right\| \\
& \quad+\frac{\delta}{c}\left|E^{\prime}(t)\right| e^{-c / 8 \delta}\left\|e^{-c Q_{3}} S_{E(t)} e^{c Q_{3}}\right\| \quad\left\|e^{-c Q_{3}} \phi_{E(t)}^{\prime}\right\| \\
& \leqq \\
& \frac{2 \delta}{c}\left|E^{\prime}(t)\right| e^{-c / 8 \delta} \cdot 8 \cdot \frac{6}{\delta} \\
& =\frac{96}{c}\left|E^{\prime}(t)\right| e^{-c / 8 \delta}
\end{aligned}
$$

by Lemmas 7 and 9 . Therefore

$$
\begin{aligned}
& |F(\eta, t)-F(\eta, 0)| \leqq|G(\eta, t)-G(\eta, 0)|+\int_{0}^{t}\left|\frac{\partial}{\partial s}(F(\eta, s)-G(\eta, s))\right| d s \\
& \leqq\left|E^{\prime}(t)\right| / 2 \delta+\left|E^{\prime}(0)\right| / 2 \delta+\int_{0}^{t}\left[\frac{8 \delta}{c} e^{-c / 8 \delta}+\left|E^{\prime \prime}(s)\right| / 2 \delta\right. \\
& \left.\quad+2\left|E^{\prime}(s)\right|^{2} / \delta^{2}+\frac{96}{c}\left|E^{\prime}(s)\right| e^{-c / 8 \delta}\right] d s,
\end{aligned}
$$

which is equivalent to (15).

The first term on the right-hand side of (15) bounds the tunneling rate. Its magnitude increases indefinitely with $t$ and does not depend on how rapidly $E(t)$ varies but only on the maximum value $\delta$ which $|E(t)|$ takes. The other terms on the right-hand side of (15) bound the impulsive reactions of the system, and are 
diminished by making $E(t)$ vary slowly. In order to make the interpretation of Theorem 12 clearer we consider a simple special case.

Corollary 13. If $E(t)=\delta B(\varepsilon t)$, where $0 \leqq B(s) \leqq 1$ if $s \leqq 1$ and $B(s)=1$ if $s>1$, then

$$
\left\|\psi(t)-e^{-i \theta(t)} \phi_{\delta}\right\| \leqq \frac{8 \delta}{c} e^{-c / 8 \delta} t+b\left(\varepsilon+\delta e^{-c / 8 \delta}\right)
$$

for all $t \geqq \varepsilon^{-1}$, where $b$ depends upon $B$ alone.

Note that in order for both terms on the right-hand side of (16) to be small when $t=\varepsilon^{-1}$ we must have $(8 \delta / c) e^{-c / 8 \delta} \ll \varepsilon \ll 1$, confirming the general comments we made in the introduction. The first term on the right-hand side of (16) yields a lower bound on the tunneling rate which is of the same general form as that given by Oppenheimer's formula [4]. The constant $c / 8$ in the exponential is of course too small, but it can be considerably increased by more careful estimation at each state, or by the application of more precise JWKB bounds to replace Lemmas 7 and 9 .

Acknowledgements. We should like to thank E. Harrell, I. Herbst, E. Lieb and B. Simon for many helpful conversations in the IAMP Conference, Berlin, 1981.

\section{References}

1. Aguilar, J., Combes, J.: A class of analytic perturbations for one-body Schrödinger Hamiltonians. Commun. Math. Phys. 22, 269-279 (1971)

2. Balslev, E., Combes, J.: Spectral properties of many-body Schrödinger operators with dilatationanalytic interactions. Commun. Math. Phys. 22, 280-294 (1971)

3. Davies, E. B.: Resonances, spectral concentration and exponential decay. Lett. Math. Phys. 1, 31--35 (1975)

4. Harrell, E., Simon, B.: The mathematical theory of resonances whose widths are exponentially small. Duke Math. J. 47, 845-902 (1980)

5. Herbst, I.: Dilation analyticity in constant electric fields, I. The two body problem. Commun. Math. Phys. 64, 279-298 (1979)

6. Kato, T.: On the adiabatic theorem of quantum mechanics. J. Phys. Soc. Jpn 5, 435-439 (1950)

7. Kato, T.: Perturbation theory for linear operators. 1st edn., Berlin, Heidelberg, New York: Springer 1966

8. O'Connor, A. J.: Exponential decay of bound state wave functions. Commun. Math. Phys. 32, 319 340 (1973)

9. Phillips, R. S.: Perturbation theory for semigroups of linear operators. Trans. Am. Math. Soc. 74, 199-221 (1954)

10. Reed, M., Simon, B.: Methods of modern mathematical physics, Vol. 2. New York: Academic Press 1975

11. Reed, M., Simon, B.: Methods of modern mathematical physics, Vol. 4. New York: Academic Press 1978

12. Simon, B.: Resonances in n-body quantum systems with dilatation-analytic potentials and the foundations of time-dependent perturbation theory. Ann. Math. 97, 247-274 (1973)

13. Simon, B.: Resonances and complex scaling: a rigorous overview. Int. J. Quantum Chem. 14, 529$542(1978)$

Communicated by B. Simon

Received October 11, 1982; in revised form December 7, 1982 
